\title{
Are Cancer-Testis Antigens Cancer Stem Cell Markers?
}

\section{Soudeh Ghafouri-Fard*}

Department of Medical Genetics, Shahid Beheshti University of Medical Sciences, Tehran 19857-17443, Iran

More than two decades have been passed from the first discovery of expression of testis specific genes in melanoma. Expression pattern of many testis specific genes has been analyzed in different tumors since then and many of them have been shown to be expressed in various histological tumor types. The term "Cancer-Testis (CT) Antigens" is now broadly used in medical literature. There are different proposed mechanisms for their aberrant expression in the process of tumorigenesis, of which epigenetic deregulation is widely accepted [1]. Their expression in syncytiotrophoblasts and cytotrophoblasts has related placentation to the process of tumorigenesis to such extent that Lloyd J. Old has called cancer "a somatic cell pregnancy" [2]. The similarities between germ line stem cells and their trophoblastic derivatives have induced the assumption that activation of normally silent germline-specific genes in cancer stem cells (CSCs) (gametic recapitulation) can cause malignancy even in the lack of mutations in oncogenes and tumor suppressor genes [3]. Since a high percentage of CT genes located on X chromosome, are expressed in the spermatogonia, which contain the spermatogonial stem cells, it has been proposed that these genes can be markers for cells having stemness features [3]. Some CT antigens such as SSX, NY-ESO-1 and N-RAGE have been shown to be expressed in undifferentiated mesenchymal stem cells, while downregulated after their differentiation [4]. In addition, the role of SSX in stem cell migration has implied a possible role for it in cancer metastasis [4]. In another study, it has been demonstrated that MAGE-A3, -A6, -A4, -A8, and GAGEs are expressed in undifferentiated human embryonic stem cells and embryoid body cells while later differentiated ones expressed only MAGE-A8 or MAGE-A4 [5].

The presence of cells having stem cell characteristics have been observed in different tumors including brain tumors [6]. These cells preserve stem cell features in tumor mass and have role in tumor proliferation and metastasis [7]. The absence or very low expression of CT genes in normal differentiated tissue indicated that CT antigen expression in tumor tissues might be restricted to cells that maintain stem cell features i.e. CSCs [7]. In a recent study of several glioblastoma stem cell isolates pre- and postdifferentiation, it has been revealed that CT antigens knock down in both cell types will lead to either changes in neural stem cell marker expression or a decreased cell density probably as a result of growth arrest or cell death [8]. This shows the importance of CT genes in stem cell phenotype.
The probable role of CT genes as stem cell marker as well as cancer biomarker has been proposed for a long time, but the evidences supporting their role as CSC marker are few. This is partly because CSC hypothesis has just recently gained attention. If future researches support this function for CT antigens, different treatment modalities including shRNA mediated silencing of their expression can be applied widely for patients. Since there is a trend to apply treatment modalities which target CSCs instead of other cancer cell populations. Because of restricted expression pattern of CT antigens in the testis and immune privileged status of the testis, current treatment options focus on induction of humoral or cytotoxic immune responses against CT antigens. In addition, pattern of CT antigens expression should be compared in normal stem cells vs. CSCs. If they are shown to be expressed widely in normal tissue stem cells, possible side effects might put their application in immunotherapeutic approaches under question.

\section{References}

1. Ghafouri-Fard S, Modarressi MH (2009) Cancer-Testis Antigens: Potential Targets for Cancer Immunotherapy. Arch Iran Med 12: 395-404.

2. Old LJ (2007) Cancer is a somatic cell pregnancy. Cancer Immun 7: 19.

3. Simpson AJ, Caballero OL, Jungbluth A, Chen YT, Old LJ (2005) Cancer/testis antigens, gametogenesis and cancer. Nat Rev Cancer 5: 615-625.

4. Cronwright G, Le Blanc K, Gotherström C, Darcy P, Ehnman M, et al. (2005) Cancer/testis antigen expression in human mesenchymal stem cells: downregulation of SSX impairs cell migration and matrix metalloproteinase 2 expression. Cancer Res 65: 2207-2215.

5. Lifantseva N, Koltsova A, Krylova T, Yakovleva T, Poljanskaya G, et al. (2011) Expression patterns of cancer-testis antigens in human embryonic stem cells and their cell derivatives indicate lineage tracks. Stem Cells Int 2011: 795239.

6. Ghafouri-Fard S, Modarressi MH (2012) Expression of cancer-testis genes in brain tumors: implications for cancer immunotherapy. Immunotherapy 4: 59-75.

7. Costa FF, Le Blanc K, Brodin B (2007) Concise review: cancer/testis antigens stem cells, and cancer. Stem Cells 25: 707-711.

8. Low J, Dowless M, Shiyanova T, Rowlinson S, Ricci-Vitiani L, et al. (2010) Knockdown of cancer testis antigens modulates neural stem cell marker expression in glioblastoma tumor stem cells. J Biomol Screen 15: 830-839.
*Corresponding author: Soudeh Ghafouri-Fard, Department of Medical Genetics, Shahid Beheshti University of Medical Sciences, Tehran 19857-17443, Iran, Tel: +98 212387 2572; Fax: +98 212387 2572; E-mail: ghafourifard@razi.tums.ac.ir

Received April 03, 2012; Accepted April 06, 2012; Published April 08, 2012

Citation: Ghafouri-Fard S (2012) Are Cancer-Testis Antigens Cancer Stem Cell Markers? Single Cell Biol 1:e104. doi:10.4172/2168-9431.1000e104

Copyright: (c) 2012 Ghafouri-Fard S. This is an open-access article distributed under the terms of the Creative Commons Attribution License, which permits unrestricted use, distribution, and reproduction in any medium, provided the original author and source are credited. 